\title{
A General Method using Geometry to Find Eigen Vectors and Eigen Values of Matrix of Size 3x3
}

Rania. B. M. Amer

Department of Engineering Mathematics and Physics, Faculty of Engineering, Zagazig University, P.O. 44519, Zagazig, Egypt

\author{
M. Afwat \\ Department of Engineering Mathematics and Physics, \\ Faculty of Engineering, Zagazig University, P.O. 44519, \\ Zagazig, Egypt
}

\begin{abstract}
In this paper, the general model symmetric matrix $3 \times 3$ is expressed. Furthermore, the general model of non symmetric matrix $3 \times 3$ is discussed. The examples are presented to verify the results.
\end{abstract}

Keywords-Lagrange multiplier ; Cardan's method ; eigen value; eigen vector.

\section{INTRODUCTION}

Eigen values are greatest importance in dynamic problems(Luenberger 1979), (Johansen 1988),(Haftka and Adelman 1989) and many engineering application (Thomson 1996). The eigenvectors (Joy 2000) denoted by $x_{i}$ and eigenvalues $\lambda_{i}$ of a any matrix $A$ that is satisfied $A x=\lambda x$. If $A$ is an $n \times n$ matrix, then $x_{i}$ is an $n \times 1$ vector, and $\lambda_{i}$ is a constant.

The matrix A has Eigen vectors and Eigen values are written as:

$\mathrm{V}=\left(\begin{array}{ccc}\mathrm{x}_{1}^{1} & \cdots & \mathrm{x}_{1}^{\mathrm{n}} \\ \vdots & \ddots & \vdots \\ \mathrm{x}_{\mathrm{n}}^{1} & \cdots & \mathrm{x}_{\mathrm{n}}^{\mathrm{n}}\end{array}\right), \mathrm{D}=\left(\begin{array}{ccc}\lambda_{1} & \cdots & 0 \\ \vdots & \ddots & \vdots \\ 0 & \cdots & \lambda_{\mathrm{n}}\end{array}\right)$

The matrix $\mathrm{V}$ is called the modal matrix of A. Since D, as a diagonal matrix, has Eigen values $\lambda_{1}, \ldots, \lambda_{n}$ which are the same as those of $\mathrm{A}$ then the matrices $\mathrm{D}$ and $\mathrm{A}$ are said to be similar. The transformation of $\mathrm{A}$ into $\mathrm{D}$ using $\mathrm{V}-1 \mathrm{~A} V=\mathrm{D}$ is said to be a similarity transformation.

\section{MATERIAL AND METHODS}

- $\quad$ To find the model matrix and the Eigen values of a symmetric matrix:

$$
\left.\begin{array}{lll}
\mathrm{a} & \mathrm{h} & \mathrm{g} \\
\mathrm{h} & \mathrm{b} & \mathrm{f} \\
\mathrm{g} & \mathrm{f} & \mathrm{c}
\end{array}\right]
$$

We express the given matrix to demonstrate a surface of second degree

$$
\left.\left[\begin{array}{llllll}
x & y & z
\end{array}\right] \begin{array}{ccc}
\mathrm{h} & \mathrm{b} & \mathrm{f} \\
\mathrm{g} & \mathrm{f} & \mathrm{c}
\end{array}\right]\left[\begin{array}{l}
\mathrm{x} \\
\mathrm{y} \\
\mathrm{z}
\end{array}\right]
$$

Then

$a x^{2}+b y^{2}+c z^{2}+2 h x y+2 f y z+2 g x z+l=0$
With center $(0,0,0)$, i.e $x_{s}=y_{s}=z_{s}=0$

We take a function: $\left(\mathrm{x}-\mathrm{x}_{\mathrm{s}}\right)^{2}+\left(\mathrm{y}-\mathrm{y}_{\mathrm{s}}\right)^{2}+\left(\mathrm{z}-\mathrm{z}_{\mathrm{s}}\right)^{2}$ and we find its extreme points on the given surface, using Lagrange multiplier $\lambda$. Then

$\varphi(\mathrm{x}, \mathrm{y}, \mathrm{z})=(\mathrm{x}-0)^{2}+(\mathrm{y}-0)^{2}+(\mathrm{z}-0)^{2}+\lambda\left(\mathrm{ax}^{2}+\right.$

$\left.b y^{2}+c z^{2}+2 h x y+2 f y z+2 g x z+l\right)$

We find:

$\frac{\partial \varphi}{\partial \mathrm{x}}=0, \frac{\partial \varphi}{\partial \mathrm{y}}=0, \frac{\partial \varphi}{\partial \mathrm{z}}=0, \frac{\partial \varphi}{\partial \lambda}=0$, then:

$-\lambda=\frac{\mathrm{x}}{\mathrm{ax}+\mathrm{hy}+\mathrm{gz}}=\frac{\mathrm{y}}{\mathrm{hx}+\mathrm{by}+\mathrm{fz}}=$

$$
=\frac{\mathrm{z}}{\mathrm{gx}+\mathrm{fy}+\mathrm{cz}} \text { and } \mathrm{ax}^{2}+\mathrm{by}^{2}+\mathrm{cz}^{2}
$$$$
+2 \mathrm{hxy}+2 \mathrm{fyz}+2 \mathrm{gxz}+\mathrm{l}=0
$$

We can find:

$h\left(x^{2}-y^{2}\right)+(b-a) x y+f x z-g y z=0$,

$g\left(x^{2}-z^{2}\right)+(c-a) x z+f x y-h y z=0$,

$f\left(y^{2}-z^{2}\right)+(c-b) y z+g x y-h x z=0$,

From equation (4), we get:

$\mathrm{x}=\frac{\mathrm{f}\left(\mathrm{y}^{2}-\mathrm{z}^{2}\right)+(\mathrm{c}-\mathrm{b}) \mathrm{yz}}{\mathrm{hz}-\mathrm{gy}}$

Multiply equation (2) by gf, Multiply equation (3) by -fh, Multiply equation (4) by gh and adding, then

$$
\begin{aligned}
& {\left[\mathrm{gfb}-\mathrm{gfa}-\mathrm{f}^{2} \mathrm{~h}+\mathrm{g}^{2} \mathrm{~h}\right] \mathrm{xy}+} \\
& {\left[\mathrm{gf}^{2}-\mathrm{fhc}+\mathrm{fha}-\mathrm{gh}^{2}\right] \mathrm{xz}+}
\end{aligned}
$$

$\left[-\mathrm{g}^{2} \mathrm{f}+\mathrm{fh}^{2}+\mathrm{cgh}-\mathrm{bgh}\right] \mathrm{yz}=0$

(6)

From equation (6), we get:

$\mathrm{x}=\frac{\left[\mathrm{g}^{2} \mathrm{f}-\mathrm{fh}^{2}-\mathrm{cgh}+\mathrm{bgh}\right] \mathrm{yz}}{\left[\mathrm{gfb}-\mathrm{gfa}-\mathrm{f}^{2} \mathrm{~h}+\mathrm{g}^{2} \mathrm{~h}\right] \mathrm{y}+\left[\mathrm{f}^{2}-\mathrm{fhc}+\mathrm{fha}-\mathrm{gh}^{2}\right] \mathrm{z}}$

From equations (5) and (7):

$\frac{f\left(y^{2}-z^{2}\right)+(c-b) y z}{h z-g y}$

$=\frac{\left[g^{2} f-f^{2}-c g h+b g h\right] y z}{\left[g f b-g f a-f^{2} h+g^{2} h\right] y+\left[g f^{2}-f h c+f h a-g h^{2}\right] z}$

The cubic equation in $\mathrm{z}$ is:

$\left[-\mathrm{f}\left(\mathrm{gf}^{2}-\mathrm{fhc}+\mathrm{fha}-\mathrm{gh}^{2}\right)\right] \mathrm{z}^{3}+\left[-\mathrm{f}\left(\mathrm{gfb}-\mathrm{gfa}-\mathrm{f}^{2} \mathrm{~h}+\right.\right.$ $\left.g^{2} h\right)+(c-b)\left(g f^{2}-f h c+f h a-g h^{2}\right)-h\left(g^{2} f-f^{2}-\right.$ cgh + bgh $)] z^{2} y+\left[f\left(g f^{2}-\right.\right.$ fhc + fha $\left.-g h^{2}\right)+$ 
$(c-b)\left(g f b-g f a-f^{2} h+g^{2} h\right)+g\left(g^{2} f-f^{2}-c g h+\right.$ bgh $)] y^{2} z+\left[f\left(g f b-g f a-f^{2} h+g^{2} h\right)\right] y^{3}=0$

(8)

$$
\begin{aligned}
& A_{0}=-f\left(g f^{2}-f h c+f h a-g h^{2}\right) \\
& A_{1}=\left[-f\left(g f b-g f a-f^{2} h+g^{2} h\right)+(c-b)\left(g f^{2}-\right.\right. \\
& \text { fhc } \left.\left.+ \text { fha }-g h^{2}\right)-h\left(g^{2} f-f^{2}-c g h+b g h\right)\right] y
\end{aligned}
$$$$
A_{2}=\left[f\left(g f^{2}-f h c+f h a-g h^{2}\right)\right.
$$

$$
\begin{aligned}
& +(c-b)\left(g f b-g f a-f^{2} h+g^{2} h\right) \\
& \left.+g\left(g^{2} f-f^{2}-c g h+b g h\right)\right] y^{2}
\end{aligned}
$$$$
A_{3}=\left[f\left(g f b-g f a-f^{2} h+g^{2} h\right)\right] y^{3}
$$

$$
\mathrm{k}=\frac{-\mathrm{A}_{1}}{3 \mathrm{~A}_{0}}
$$

Then the cubic equation (8) can be written in its reduced form: $\mathrm{A}_{0} \mathrm{z}^{3}+\left(\mathrm{A}_{2}+\mathrm{kA}_{1}\right) \mathrm{z}+\left(\mathrm{A}_{3}+\mathrm{kA}_{2}+\frac{2}{3} \mathrm{k}^{2} \mathrm{~A}_{1}\right)=0$

For Cardan's method:

$$
\begin{gathered}
M=\frac{A_{3}+k A_{2}+k^{2} A_{1}+k^{3} A_{0}}{2 A_{0}}=\frac{A_{3}+k A_{2}+\frac{2}{3} k^{2} A_{1}}{2 A_{0}}, \\
N=\frac{A_{2}+2 \mathrm{kA}_{1}+3 k^{2} A_{0}}{3 A_{0}}=\frac{A_{2}+k A_{1}}{3 A_{0}} \\
r=S Q R\left[M^{2}+A B S\left(M^{3}+N^{3}\right)\right] \\
L=\frac{-M}{r}
\end{gathered}
$$

if $\mathrm{L}<0$, then $\theta_{0}=180-\arccos (-\mathrm{L})=\arccos (\mathrm{L})$,

if $L>0$, then $\theta_{0}=\arccos (L)$,

$$
\begin{gathered}
\theta_{1}=\frac{\theta_{0}}{3} \\
\mathrm{R}=2 \sqrt[3]{\mathrm{r}} \\
\mathrm{z}_{1}=\mathrm{R} \cos \theta_{1}+\mathrm{k}, \\
\mathrm{z}_{2}=\mathrm{R} \cos \left(\theta_{1}+120\right)+\mathrm{k} \\
\mathrm{z}_{3}=\mathrm{R} \cos \left(\theta_{1}+240\right)+\mathrm{k} .
\end{gathered}
$$

By the following relation

$$
x_{i}=\frac{f\left(y_{i}^{2}-z_{i}^{2}\right)+(c-b) y_{i} z_{i}}{h z_{i}-g y_{i}} ; i=1,2,3
$$

To find $\mathrm{x}_{1}, \mathrm{x}_{2}, \mathrm{x}_{3}$ using $\mathrm{z}_{1}, \mathrm{z}, \mathrm{z}_{3}$

$$
\text { The model matrix is } \left.\begin{array}{ccc}
\mathrm{x}_{1} & \mathrm{x}_{2} & \mathrm{x}_{3} \\
\mathrm{y}_{1} & \mathrm{y}_{2} & \mathrm{y}_{3} \\
\mathrm{z}_{1} & \mathrm{z}_{2} & \mathrm{z}_{3}
\end{array}\right]
$$

To find the Eigen values:

$$
\left.\left[\begin{array}{llllll}
\mathrm{a}-\lambda & \mathrm{h} & \mathrm{g}
\end{array}\right] \begin{array}{ccc}
\mathrm{x}_{1} & \mathrm{x}_{2} & \mathrm{x}_{3} \\
\mathrm{y}_{1} & \mathrm{y}_{2} & \mathrm{y}_{3} \\
\mathrm{z}_{1} & \mathrm{z}_{2} & \mathrm{z}_{3}
\end{array}\right]=\left[\begin{array}{l}
0 \\
0 \\
0
\end{array}\right]
$$

Then

$$
\begin{aligned}
& \lambda_{1}=\frac{\mathrm{ax}_{1}+\mathrm{hy}_{1}+\mathrm{gz}_{1}}{\mathrm{x}_{1}}, \\
& \lambda_{2}=\frac{\mathrm{ax}_{2}+\mathrm{hy}_{2}+\mathrm{gz}_{2}}{\mathrm{x}_{2}}, \\
& \lambda_{3}=\frac{\mathrm{ax}_{3}+\mathrm{hy}_{3}+\mathrm{gz}_{3}}{\mathrm{x}_{3}}
\end{aligned}
$$

Example (1):

Find the model matrix and Eigen values of the

$$
\left.\operatorname{matrix} \begin{array}{ccc}
11 & -6 & 2 \\
2 & 10 & -4 \\
2 & -4 & 6
\end{array}\right]
$$

Solution:

$$
a=11, b=10, c=6, h=-6, g=2, f=-4 \neq 0,
$$

Then

$\mathrm{A}_{0}=320, \mathrm{~A}_{1}=480 \mathrm{y}, \mathrm{A}_{2}=-480 \mathrm{y}^{2}, \mathrm{~A}_{3}=$

$-320 \mathrm{y}^{3}, \mathrm{k}=-0.5 \mathrm{y}$

$\mathrm{M}=\frac{-320 \mathrm{y}^{3}+0.5 * 480 \mathrm{y}^{3}+\frac{2}{3}(0.5)^{2} 480 \mathrm{y}^{3}}{2 * 320}=0$,

$\mathrm{N}=\frac{\mathrm{A}_{2}+2 \mathrm{kA}_{1}+3 \mathrm{k}^{2} \mathrm{~A}_{0}}{3 \mathrm{~A}_{0}}$

$=\frac{-480 \mathrm{y}^{2}+2 *-0.5 * 480 \mathrm{y}^{2}+3 *(-0.5)^{2} * 320 \mathrm{y}^{2}}{3 * 320}$

$=-0.7500 \mathrm{y}^{2}$

$\mathrm{r}=0.6495 \mathrm{y}^{3}, \mathrm{~L}=0, \theta_{0}=\arccos \mathrm{L}=\frac{\pi}{2}, \theta_{1}=\frac{\theta_{0}}{3}=\frac{\pi}{6}, \mathrm{R}=$ $2 \sqrt[3]{\mathrm{r}}=1.7321 \mathrm{y}$

Then

$$
\mathrm{z}_{1}=\mathrm{y}_{1}, \mathrm{z}_{2}=-2 \mathrm{y}_{2}, \mathrm{z}_{3}=-0.5 \mathrm{y}_{3} \text {, }
$$

$\mathrm{x}_{1}=\frac{\mathrm{f}\left(\mathrm{y}_{1}^{2}-\mathrm{z}_{1}^{2}\right)+(\mathrm{c}-\mathrm{b}) \mathrm{y}_{1} \mathrm{z}_{1}}{\mathrm{hz_{1 }}-\mathrm{gy}_{1}}=0.5 \mathrm{y}_{1}$

$x_{2}=\frac{f\left(y_{2}^{2}-z_{2}^{2}\right)+(c-b) y_{2} z_{2}}{h z_{2}-g y_{2}}=2 y_{2}$,

$x_{3}=\frac{f\left(y_{3}^{2}-z_{3}^{2}\right)+(c-b) y_{3} z_{3}}{h z_{3}-g y_{3}}=-y_{3}$,

Then the model matrix is:

$$
\left.\begin{array}{ccc}
\mathrm{x}_{1} & \mathrm{x}_{2} & \mathrm{x}_{3} \\
\mathrm{y}_{1} & \mathrm{y}_{2} & \mathrm{y}_{3} \\
\mathrm{z}_{1} & \mathrm{z}_{2} & \mathrm{z}_{3}
\end{array}\right]=\left[\begin{array}{ccc}
0.5 & 2 & -1 \\
1 & 1 & 1 \\
1 & -2 & -0.5 \\
\lambda_{1} & =\frac{\mathrm{ax}_{1}+\mathrm{hy}_{1}+\mathrm{gz}_{1}}{\mathrm{x}_{1}}=3 \\
\lambda_{2} & =\frac{\mathrm{ax}_{2}+\mathrm{hy}_{2}+\mathrm{gz}_{2}}{\mathrm{x}_{2}}=6 \\
\lambda_{3} & =\frac{\mathrm{ax}_{3}+\mathrm{hy}_{3}+\mathrm{gz}_{3}}{\mathrm{x}_{3}}=18
\end{array}\right.
$$

To check the results:

- By using Maple program

$>$ with (LinearAlgebra) :

$\mathrm{E}:=\langle\langle 11,-6,2\rangle\langle\langle-6,10,-4\rangle\langle(2,-4,6\rangle\rangle$

$$
\left[\begin{array}{rrr}
11 & -6 & 2 \\
-6 & 10 & -4 \\
2 & -4 & 6
\end{array}\right]
$$

Eigenvalues $($ E, output $=$ 'list' $)$

$[3,6,18]$

$v, e:=$ Eigenvectors $(E)$

$$
\left[\begin{array}{r}
6 \\
18 \\
3
\end{array}\right],\left[\begin{array}{ccc}
-1 & 2 & \frac{1}{2} \\
-\frac{1}{2} & -2 & 1 \\
1 & 1 & 1
\end{array}\right]
$$

- $\quad$ By using Matlab program

$$
\begin{aligned}
>>A & =\left[\begin{array}{llllll}
11 & -6 & 2 ;-6 & 10 & -4 ; 2 & -4
\end{array}\right] \\
& > \\
& {[\mathrm{V}, \mathrm{D}]=\operatorname{eig}(\mathrm{A}) }
\end{aligned}
$$




$$
\begin{array}{cccc}
\mathrm{V}= & 0.3333 & -0.6667 & 0.6667 \\
0.6667 & -0.3333 & -0.6667 \\
0.6667 & 0.6667 & 0.3333 \\
\multicolumn{4}{c}{\mathrm{D}=} \\
3.0000 & 0 & 0 \\
0 & 6.0000 & 0 \\
0 & 0 & 18.0000
\end{array}
$$

- To find the model matrix and the Eigen values of a given non symmetric matrix:

$$
A=\left[\begin{array}{lll}
a & d & e \\
f & b & k \\
g & h & c
\end{array}\right]
$$

We express the given matrix to demonstrate a surface of second degree

$$
\left.\mathrm{Q}=\left[\begin{array}{lllll}
\mathrm{x} & \mathrm{y} & \mathrm{z}
\end{array}\right] \begin{array}{ccc}
\mathrm{f} & \mathrm{b} & \mathrm{k} \\
\mathrm{g} & \mathrm{h} & \mathrm{c}
\end{array}\right]\left[\begin{array}{l}
\mathrm{x} \\
\mathrm{y} \\
\mathrm{z}
\end{array}\right]
$$
Then

$Q=a x^{2}+b y^{2}+c z^{2}+(d+f) x y+(k+h) y z+(e+g) x z+l=0$

With center $(0,0,0)$, i.e $x_{s}=y_{s}=z_{s}=0$

We take a function: $\left(x-x_{s}\right)^{2}+\left(y-y_{s}\right)^{2}+\left(z-z_{s}\right)^{2}$ and we

find its extreme points on the given surface, using Lagrange multiplier $\lambda$. Then

$$
\begin{aligned}
& \varphi(\mathrm{x}, \mathrm{y}, \mathrm{z})=\mathrm{x}^{2}+\mathrm{y}^{2}+\mathrm{z}^{2}+\lambda\left(\mathrm{ax}^{2}+\mathrm{by}^{2}+\mathrm{cz}^{2}+\right. \\
& (d+f) x y+(k+h) y z+(e+g) x z+l) \\
& \text { We find: } \\
& \frac{\partial \varphi}{\partial \mathrm{x}}=0, \frac{\partial \varphi}{\partial \mathrm{y}}=0, \frac{\partial \varphi}{\partial \mathrm{z}}=0, \frac{\partial \varphi}{\partial \lambda}=0 \text {, then: } \\
& -\lambda=\frac{2 \mathrm{x}}{2 \mathrm{ax}+(\mathrm{d}+\mathrm{f}) \mathrm{y}+(\mathrm{e}+\mathrm{g}) \mathrm{z}} \\
& =\frac{2 y}{(d+f) x+2 b y+(k+h) z}= \\
& =\frac{2 z}{(e+g) x+(k+h) y+2 c z} \text { and } a^{2} \\
& +b^{2}+c^{2}+(d+f) x y+(k+h) y z \\
& +(\mathrm{e}+\mathrm{g}) \mathrm{xz}+\mathrm{l}=0 \\
& \text { We can find: }
\end{aligned}
$$

From equation (4), we get:

$$
\mathrm{x}=\frac{(\mathrm{k}+\mathrm{h})\left(\mathrm{y}^{2}-\mathrm{z}^{2}\right)+2(\mathrm{c}-\mathrm{b}) \mathrm{yz}}{(\mathrm{d}+\mathrm{f}) \mathrm{z}-(\mathrm{e}+\mathrm{g}) \mathrm{y}}
$$

Multiply equation (2) by $(\mathrm{e}+\mathrm{g})(\mathrm{k}+\mathrm{h})$, multiply equation (3)

by $-(\mathrm{k}+\mathrm{h})(\mathrm{d}+\mathrm{f})$, multiply equation $(4)$ by $(\mathrm{e}+\mathrm{g})(\mathrm{d}+\mathrm{f})$ and adding, then

$$
\begin{gathered}
{\left[2(b-a)(e+g)(k+h)-(d+f)(h+k)^{2}+(d+f)(e+g)^{2}\right]} \\
{\left[(e+g)(k+h)^{2}-2(c-a)(d+f)(k+h)-(e+g)(d+f)^{2}\right] x z+} \\
{\left[-(k+h)(e+g)^{2}+(k+h)(d+f)^{2}+\right.} \\
2(c-b)(e+g)(d+f)] y z=0
\end{gathered}
$$

$\frac{(k+h)\left(y^{2}-z^{2}\right)+2(c-b) y z}{(d+f) z-(e+g) y}=$

$$
(\mathrm{d}+\mathrm{f}) \mathrm{z}-(\mathrm{e}+\mathrm{g}) \mathrm{y}
$$

$\left[(\mathrm{k}+\mathrm{h})(\mathrm{e}+\mathrm{g})^{2}-(\mathrm{k}+\mathrm{h})(\mathrm{d}+\mathrm{f})^{2}-2(\mathrm{c}-\mathrm{b})(\mathrm{e}+\mathrm{g})(\mathrm{d}+\mathrm{f})\right] \mathrm{yz}$

$\left[2(b-a)(e+g)(k+h)-(d+f)(h+k)^{2}+(d+f)(e+g)^{2}\right] y+\left[(e+g)(k+h)^{2}-2(c-a)(d+f)(k+h)-(e\right.$ Then

$$
\begin{aligned}
& \mathrm{A}_{3} \stackrel{(10)}{=}(\mathrm{k}+\mathrm{h}) \\
& A_{0}=-(k+h)\left[(e+g)(k+h)^{2}-2(c-a)(d+f)(k+h)\right. \\
& \left.-(e+g)(d+f)^{2}\right] \\
& A_{1}=\left[-(k+h)\left[2(b-a)(e+g)(k+h)-(d+f)(h+k)^{2}\right.\right. \\
& \left.+(d+f)(e+g)^{2}\right] \\
& +2(\mathrm{c}-\mathrm{b})\left[(\mathrm{e}+\mathrm{g})(\mathrm{k}+\mathrm{h})^{2}\right. \\
& -2(c-a)(d+f)(k+h) \\
& \left.-(\mathrm{e}+\mathrm{g})(\mathrm{d}+\mathrm{f})^{2}\right] \\
& -(\mathrm{d}+\mathrm{f})\left[(\mathrm{k}+\mathrm{h})(\mathrm{e}+\mathrm{g})^{2}\right. \\
& -(k+h)(d+f)^{2}-2(c-b)(e+g)(d \\
& +\mathrm{f})] \mathrm{y} \\
& A_{2}=\left[( k + h ) \left[(e+g)(k+h)^{2}-2(c-a)(d+f)(k+h)\right.\right. \\
& \left.-(e+g)(d+f)^{2}\right] \\
& +2(\mathrm{c}-\mathrm{b})[2(\mathrm{~b}-\mathrm{a})(\mathrm{e}+\mathrm{g})(\mathrm{k}+\mathrm{h}) \\
& \left.-(d+f)(h+k)^{2}+(d+f)(e+g)^{2}\right] \\
& +(\mathrm{e}+\mathrm{g})\left[(\mathrm{k}+\mathrm{h})(\mathrm{e}+\mathrm{g})^{2}\right. \\
& -(k+h)(d+f)^{2}-2(c-b)(e+g)(d \\
& +\mathrm{f})]] \mathrm{y}^{2} \\
& \begin{array}{l}
2(b-a)(e+g)(k+h)-( \\
\left.\left.+(d+f)(e+g)^{2}\right]\right] y^{3}
\end{array} \\
& \mathrm{k}=\frac{-\mathrm{A}_{1}}{3 \mathrm{~A}_{0}}
\end{aligned}
$$

Then the cubic equation (8) can be written in its reduced form:

$$
A_{0} z^{3}+\left(k A_{1}+A_{2}\right) z+\left(A_{3}+k A_{2}+\frac{2}{3} k^{2} A_{1}\right)
$$

Using Cardan's method we find the three roots of equation (9)

$$
z_{1}^{\prime}, z_{2}^{\prime}, z_{3}^{\prime}
$$

We obtain the three roots of equation (8):

$$
\begin{aligned}
& \mathrm{z}_{1}=\mathrm{z}_{1}^{\prime}+\mathrm{k}, \quad \mathrm{z}_{2}=\mathrm{z}_{2}^{\prime}+\mathrm{k}, \quad \mathrm{z}_{3}=\mathrm{z}_{3}^{\prime}+\mathrm{k}, \\
& \text { By the following relation } \\
& \mathrm{x}=\frac{(\mathrm{k}+\mathrm{h})\left(\mathrm{y}_{\mathrm{i}}^{2}-\mathrm{z}_{\mathrm{i}}^{2}\right)+2(\mathrm{c}-\mathrm{b}) \mathrm{y}_{\mathrm{i}} \mathrm{z}_{\mathrm{i}}}{(\mathrm{d}+\mathrm{f}) \mathrm{z}_{\mathrm{i}}-(\mathrm{e}+\mathrm{g}) \mathrm{y}_{\mathrm{i}}} ; \mathrm{i}=1,2,3
\end{aligned}
$$

To find $\mathrm{x}_{1}, \mathrm{x}_{2}, \mathrm{x}_{3}$ using $\mathrm{z}_{1}, \mathrm{z}, \mathrm{z}_{3}$

$$
\text { The model matrix is } V=\left[\begin{array}{lll}
\mathrm{x}_{1} & \mathrm{x}_{2} & \mathrm{x}_{3} \\
\mathrm{y}_{1} & \mathrm{y}_{2} & \mathrm{y}_{3} \\
\mathrm{z}_{1} & \mathrm{z}_{2} & \mathrm{z}_{3}
\end{array}\right]
$$

To find the Eigen values:

$$
\left.\left[\begin{array}{llll}
a-\lambda & (\mathrm{d}+\mathrm{f}) / 2 & (\mathrm{e}+\mathrm{g}) / 2
\end{array}\right] \begin{array}{lll}
\mathrm{x}_{1} & \mathrm{x}_{2} & \mathrm{x}_{3} \\
\mathrm{y}_{1} & \mathrm{y}_{2} & \mathrm{y}_{3} \\
\mathrm{z}_{1} & \mathrm{z}_{2} & \mathrm{z}_{3}
\end{array}\right]=\left[\begin{array}{lll}
0 & 0 & 0
\end{array}\right]
$$$$
\text { Then }
$$$$
\lambda_{i}=\frac{a x_{i}+\left(\frac{d+f}{2}\right) y_{i}+\left(\frac{e+g}{2}\right) z_{i}}{x_{i}}, i=1,2,3
$$

From equation (6), we get:

$=\frac{\left[(k+h)(e+g)^{2}-(k+h)(d+f)^{2}-2(c-b)(e+g)(d+f)\right] y z}{\left[2(b-a)(e+g)(k+h)-(d+f)(h+k)^{2}+(d+f)(e+g)^{2}\right] y+\left[(e+g)(k+h)^{2}-2(c-a)(d+f)(k+h)-(e+g)(d+f)^{2}\right] z}$

From equations (5) and (6): 


\section{Example(2):}

Find the model matrix and the Eigen values of non symmetric matrix

Solution:

$$
A=\left[\begin{array}{ccc}
11 & -4 & 1 \\
-8 & 10 & -6 \\
3 & -2 & 6
\end{array}\right]
$$

$$
a=11, b=10, c=6, d=-4, e=1, f=-8, k=-6, g
$$$$
=3, \mathrm{~h}=-2
$$

Since

$$
\begin{gathered}
\mathrm{A}_{0}=5120 \\
\mathrm{~A}_{1}=7680 \mathrm{y} \\
\mathrm{A}_{2}=-7680 \mathrm{y}^{2} \\
\mathrm{~A}_{3}=-5120 \mathrm{y}^{3} \\
\mathrm{k}=\frac{-\mathrm{A}_{1}}{3 \mathrm{~A}_{0}}=-0.5000 \mathrm{y} \\
\mathrm{M}=\frac{\mathrm{A}_{3}+\mathrm{kA}_{2}+\mathrm{k}^{2} \mathrm{~A}_{1}+\mathrm{k}^{3} \mathrm{~A}_{0}}{2 \mathrm{~A}_{0}}=\frac{\mathrm{A}_{3}+\mathrm{kA}_{2}+\frac{2}{3} \mathrm{k}^{2} \mathrm{~A}_{1}}{2 \mathrm{~A}_{0}}=0 \\
\mathrm{~N}=\frac{\mathrm{A}_{2}+2 \mathrm{kA}_{1}+3 \mathrm{k}^{2} \mathrm{~A}_{0}}{3 \mathrm{~A}_{0}}=\frac{\mathrm{A}_{2}+\mathrm{kA}}{3 \mathrm{~A}_{0}} \\
=-0.7500 \mathrm{y}^{2} \\
\left.\mathrm{r}=\mathrm{SQR}\left[\mathrm{M}^{2}+\mathrm{ABS}^{2}+\mathrm{M}^{3}\right)\right]=0.6495 \mathrm{y}^{3} \\
\Delta=\mathrm{M}^{2}+\mathrm{N}^{3}=-0.4219<0 \\
\mathrm{Then}_{\text {the three roots are real }} \\
\mathrm{L}=\frac{-\mathrm{M}}{\mathrm{r}}=0
\end{gathered}
$$

if $\mathrm{L}<0$, then $\theta_{0}=180-\arccos (-\mathrm{L})=\arccos (\mathrm{L})$, if $\mathrm{L}>0$, then $\theta_{0}=\arccos (\mathrm{L})=90$,

$$
\begin{gathered}
\theta_{1}=\frac{\theta_{0}}{3}=30, \\
\mathrm{R}=2 \sqrt[3]{\mathrm{r}}=1.7321 \mathrm{y} \\
\mathrm{z}_{1}=\mathrm{R} \cos \theta_{1}+\mathrm{k}=\mathrm{y}_{1}, \\
\mathrm{z}_{2}=\mathrm{R} \cos \left(\theta_{1}+120\right)+\mathrm{k}=-2 \mathrm{y}_{2}, \\
\mathrm{z}_{3}=\mathrm{R} \cos \left(\theta_{1}+240\right)+\mathrm{k}=-0.5 \mathrm{y}_{3} . \\
\mathrm{x}_{1}=\frac{(\mathrm{k}+\mathrm{h})\left(\mathrm{y}_{1}^{2}-\mathrm{z}_{1}^{2}\right)+2(\mathrm{c}-\mathrm{b}) \mathrm{y}_{1} \mathrm{z}_{1}}{(\mathrm{~d}+\mathrm{f}) \mathrm{z}_{1}-(\mathrm{e}+\mathrm{g}) \mathrm{y}_{1}}=0.5 \mathrm{y}_{1} \\
\mathrm{x}_{2}=2 \mathrm{y}_{2}, \\
\mathrm{x}_{3}=-\mathrm{y}_{3}
\end{gathered}
$$

Then the model matrix is:

$$
\begin{gathered}
\mathrm{V}=\left[\begin{array}{lll}
\mathrm{x}_{1} & \mathrm{x}_{2} & \mathrm{x}_{3} \\
\mathrm{y}_{1} & \mathrm{y}_{2} & \mathrm{y}_{3} \\
\mathrm{z}_{1} & \mathrm{z}_{2} & \mathrm{z}_{3}
\end{array}\right]=\left[\begin{array}{ccc}
0.5 & 2 & -1 \\
1 & 1 & 1 \\
1 & -2 & -0.5
\end{array}\right], \\
\lambda_{\mathrm{i}}=\frac{\mathrm{ax}_{\mathrm{i}}+\left(\frac{\mathrm{d}+\mathrm{f}}{2}\right) \mathrm{y}_{\mathrm{i}}+\left(\frac{\mathrm{e}+\mathrm{g}}{2}\right) \mathrm{z}_{\mathrm{i}}}{\mathrm{x}_{\mathrm{i}}}, \mathrm{i}=1,2,3 \\
\text { So }
\end{gathered}
$$

$$
\begin{aligned}
& \lambda_{1}=3, \\
& \lambda_{2}=6, \\
& \lambda_{3}=18
\end{aligned}
$$

\section{CONCLUSION}

The examples is discussed in the above sections provide us a way to generalize the model matrix for non symmetric matrix. $\left[\begin{array}{lll}\text { The } & \text { d } & \text { e } \\ \mathrm{f} & \mathrm{b} & \mathrm{k} \\ \mathrm{g} & \mathrm{h} & \mathrm{c}\end{array}\right]$ is equivelant to the symmetric matrix $\left[\begin{array}{ccc}\mathrm{a} & \mathrm{h} & \mathrm{g} \\ \mathrm{h} & \mathrm{b} & \mathrm{f} \\ \mathrm{g} & \mathrm{f} & \mathrm{k}\end{array}\right]$, Where $h=\frac{f+d}{2}, g=\frac{e+g}{2}, f=\frac{k+h}{2}$.

\section{REFERENCES}

[1] Haftka, R. T. and H. M. Adelman (1989). "Recent developments in structural sensitivity analysis." Structural optimization 1(3): 137-151.

[2] Johansen, S. (1988). "Statistical analysis of cointegration vectors." Journal of economic dynamics and control 12(2-3): 231-254.

[3] Joy, K. (2000). "On-Line Geometric Modeling Notes. Eigenvalues and eigenvectors." Computer Science Department, University of California, Davis.

[4] Luenberger, D. G. (1979). Introduction to dynamic systems: theory, models, and applications, Wiley New York.

[5] Thomson, W. (1996). Theory of vibration with applications, CRC Press. 